\title{
Isoeugenol Affects Expression Pattern of Conidial Hydrophobin Gene RodA and Transcriptional Regulators MedA and SomA Responsible for Adherence and Biofilm Formation in Aspergillus fumigatus
}

Lovely Gupta

Amity University

Pooja Sen

Amity University

Asish K Bhattacharya

CSIR-National Chemical Laboratory: National Chemical Laboratory CSIR

Pooja Vijayaraghavan ( $\square$ vrpooja@amity.edu )

Amity University https://orcid.org/0000-0001-5943-9462

\section{Research Article}

Keywords: Aspergillus fumigatus, isoeugenol, virulence, hydrophobicity, transcription regulators

Posted Date: October 27th, 2021

DOI: https://doi.org/10.21203/rs.3.rs-1016518/v1

License: (9) This work is licensed under a Creative Commons Attribution 4.0 International License.

Read Full License

Version of Record: A version of this preprint was published at Archives of Microbiology on March 22nd, 2022. See the published version at https://doi.org/10.1007/s00203-022-02817-w. 


\section{Abstract}

Aspergillus fumigatus is one of the major pathogenic fungal species, causing life-threatening infections. Due to a limited spectrum of available antifungals, exploration of new drug targets as well as potential antifungal molecules has become pertinent. Rodlet layer plays an important role in adherence of fungal conidia to hydrophobic cell surfaces in host, which also leads to $A$. fumigatus biofilm formation, contributing factor to fungal pathogenicity. From decades, natural sources have been known for the development of new active molecules. The present study investigates effect of isoeugenol on genes responsible for hydrophobins (RodA), adhesion as well as biofilm formation (MedA and SomA) of $A$. fumigatus. Minimum inhibitory concentrations ( $\mathrm{MIC}$ and $\mathrm{IC}_{50}$ ) of isoeugenol against $A$. fumigatus were determined using broth microdilution assay. The $\mathrm{IC}_{50}$ results showed reduced hydrophobicity and biofilm formation after treatment with the compound and electron micrograph data corroborated these findings. The qRT-PCR showed a significant downregulation of genes RodA, MedA, SomA and pksP involved in hydrophobicity and biofilm formation. SwissADME studies potentiated drug-like propensity for isoeugenol which formed four hydrogen bonds with low binding energy $(-4.54 \mathrm{Kcal} / \mathrm{mol})$ at the catalytic site of RodA protein studied via AutoDock4. Hence, the findings conclude that isoeugenol inhibits conidial hydrophobicity and biofilm formation of $A$. fumigatus and further investigations are warranted in this direction.

\section{Introduction}

Aspergillosis has become the second most common invasive fungal infection in patients with weak immune response, particularly neutropenic patients with hematopoietic stem cell transplantation, solid organ transplantation, and those receiving chemotherapy (Abastabar et al. 2019). Recently, COVID-19 associated pulmonary aspergillosis was reported as a lethal complication affecting critically ill patients with acute respiratory distress syndrome (Borman et al. 2020). This life-threatening fungal disease is caused by ubiquitous filamentous mold, Aspergillus fumigatus that produces large number of small size airborne spores capable of surviving under harsh biotic as well as abiotic stress conditions (Kasprzyk 2008). The mainstay antifungal drugs against $A$. fumigatus infections mainly target fungal cell membrane components and enzymes involved in its biosynthesis. Despite availability of the antifungal drugs, there are many limitations, including poor oral bioavailability, narrow therapeutic indices and emerging drug resistance resulting from their excessive use (Hagiwara et al. 2016; Mota Fernandes et al. 2021). Thus, the discovery of a novel antifungal with broad-spectrum activity, low toxicity, minimal side effects and enhanced bioavailability is highly warranted (Brauer et al. 2019).

A. fumigatus possesses multiple virulence determinants like, conidial cell surface molecules (rodlet layer, DHN-melanin, pathogen associated molecular patterns (PAMPs)), secondary metabolite gliotoxin, thermotolerance, biofilm formation, oxidative stress components (Abad et al. 2010; Croft et al. 2016; Voltersen et al. 2018; Gupta et al. 2021). Adherence to a host cell represents the most crucial step of fungal pathogenesis (Lin et al. 2015). A. fumigatus spores adhere to the lung epithelial cells of the host upon direct inhalation, with subsequent germination and hyphal outgrowth representing the first stage of 
infection (Escobar et al. 2016). Its conidia possess a surface protein coat known as rodlet layer, which masks their recognition by the host immune response (Aimanianda et al. 2009). The rodlet layer is a thin coating of regularly arranged hydrophobin proteins covalently bound to conidial cell wall through glycosylphosphatidylinositol (GPI)-remnants. The rodlet layer is composed of multiple hydrophobin (Rod) proteins encoded by their respective genes $(\operatorname{Rod} A-G)$ (Cerqueira et al. 2014). Among all the Rod proteins, RodA is the only essential hydrophobin responsible for outer layer permeability, stability, hydrophobicity and immune-inertia of the conidial cell wall surface (Valsecchi et al. 2019). Deletion of RodA gene modifies the properties of the conidial cell wall surface (Valsecchi et al. 2017). The decrease in surface RodA rodlets results in increased phagocytosis of $A$. fumigatus spores (Dagenais et al. 2010) [17]. It has been reported that adherence of conidia to the host epithelial lining is reduced due to loss of conidial hydrophobin RodA (Croft et al. 2016). RodB was also found to be present in conidia but disruption of $\operatorname{rod} B$ gene did not affect the rodlet formation (Paris et al. 2003). The significance of other hydrophobins (RodC-G) in A. fumigatus is still unknown (Valsecchi et al. 2017). According to Gravelat et al (2010), the preliminary expression analysis of putative $A$. fumigatus adhesins also indicated down-regulation of the expression of a GPI-anchored protein Afu3g00880.

Regulatory proteins governing conidiation, adherence, cell wall homeostasis and biofilm formation in $A$. fumigatus includes Medusa (MedA) and transcription factor SomA. MedA has been reported to control conidiation and biofilm formation along with expression of downstream adhesin genes in A. fumigatus (Abdallah et al. 2012). It is required for adhesion to several host surfaces like pulmonary epithelial cell, endothelial cells and fibronectin. The $\Delta M e d A$ mutant studies in Galleria mellonella as well as mammalian models revealed that the mutants were pigment less with reduced virulence (Gravelat et al. 2010). Also, conidiation was reported to be affected in MedA deletion strain of $A$. fumigatus. According to Gravelat et al (2010), in $\triangle M e d A$ strain delayed conidial pigmentation was observed along with reduced gene expression of six gene cluster involved in dihydroxynaphthalene (DHN)-melanin biosynthesis as compared to wild-type. In DHN-melanin biosynthesis, $p k s P$ gene deletion leads to loss of melanin which results in modification in the $A$. fumigatus cell wall (Bayry et al. 2014). The $p k s P$ mutant produces hydrophilic conidia due to deposition of glycoproteins on their conidial cell wall. Under normal conditions, transcriptional regulator SomA functions along with its co-regulator PtaB, and plays a critical role in conidial adhesion and biofilm formation. The galactosaminogalactan (GAG) is an important component of fungal cell wall that are important in fungal adhesion and also one of the components in extracellular matrix (ECM) formation. It has been reported that the galactose and galactosamine levels reduce in $\triangle$ SomA mutants (Lin et al. 2015). SomA binds to the promoter site of GAG biosynthetic genes adg3 and ega3 (Chen et al. 2020) and affects its downstream gene expression. It also regulates the expression of genes MedA, StuA, uge3 of A. fumigatus conidiation and adhesion. According to Lin et al (2015), SomA also affects the expression of RodA. The cell wall homeostasis in A. fumigatus is somewhat interlinked with the hydrophobins (RodA), and adhesion gene regulators (MedA and SomA).

Natural compounds; a rich source of novel prototypes of antifungals can be used to solve the problems associated such as nephrotoxicity, hepatotoxicity, bronchospasm etc. with the use of current antifungals available for the treatment of $A$. fumigatus infections (invasive aspergillosis, broncho pulmonary 
aspergillosis etc) (Aldholmi et al. 2019). Isoeugenol is a phenylpropanoid and isomer of eugenol which is generally found in plants like Syzygium aromaticum, Myristica fragrans, Cinnamomum verum, Ocimum tenuiflorum, Piper nigrum (Khalil et al. 2017). It has been reported to have antimicrobial, antioxidant, antitumor activities (Peixoto et al. 2017; Zarlaha et al. 2014). Antifungal activity of isoeugenol has been reported against various Aspergillus spp. (Campaniello et al. 2010; Pizzolitto et al. 2015; Mutlu-Ingok et al. 2020). However, the mechanism of inhibitory action of isoeugenol against $A$ fumigatus is yet to be explored. Hence, in the present study, isoeugenol was investigated for its antifungal activity targeting conidial hydrophobicity and transcription regulators MedA and SomA involved in adherence, conidiation and biofilm formation in A. fumigatus.

\section{Material And Methods}

Fungal strain, culture maintenance and inoculum preparation. A. fumigatus (ATCC-46645; a gift from Prof. Axel Brakhage, Department of Molecular and Applied Microbiology, Leibniz Institute for Natural Product Research and Infection Biology-HKl, Germany), was sub-cultured on Czapek Dox agar (CzA) at 28 $\pm 2^{\circ} \mathrm{C}$ for 4 days. Briefly, $A$. fumigatus spores were harvested in sterile phosphate buffered saline (1xPBS) supplemented with $0.05 \%$ Tween 20 . The conidial suspension was adjusted to the final concentration of $5 \times 10^{4}$ conidia/ml according to CLSI M38-A2 reference method (Alexander and Clinical and Laboratory Standards Institute 2017).

Isoeugenol $\left(\mathrm{C}_{10} \mathrm{H}_{12} \mathrm{O}_{2}\right)$ was procured from Sigma-Aldrich (India). The compound was solubilised in $100 \%$ dimethyl sulfoxide (DMSO) to make a stock concentration of $100 \mathrm{mg} / \mathrm{mL}$. For working solution, stock solution was further diluted in Czapek Dox broth (CzB). It was ensured that the final concentration of DMSO did not exceed an amount that had any detectable effect in assays (Szumilak et al. 2017).

In-vitro antifungal activity. Minimum inhibitory concentration (MIC) and inhibitory concentration-50 (IC $\left.{ }_{50}\right)$ was calculated according to the CLSI M38-A2 broth microdilution method for filamentous fungi (Alexander and Clinical and Laboratory Standards Institute 2017). The experiment was carried out in triplicates in a 96-well polystyrene flat bottom plate (Tarsons, India). Isoeugenol was dissolved in DMSO and DMSO kept as solvent control. Two-fold dilutions of isoeugenol and DMSO (solvent control) were prepared in $\mathrm{CzB}$. Conidial suspension $(100 \mu \mathrm{L})$ was added to each well except negative control. The plates were incubated statically for 4 days at $28 \pm 2^{\circ} \mathrm{C}$. The MIC and IC ${ }_{50}$ are defined as the lowest concentration of the compound, which inhibit $100 \%$ and $50 \%$ of microbial growth, respectively (Alexander and Clinical and Laboratory Standards Institute 2017). The results were expressed in microgram per milliliters $(\mu \mathrm{g} / \mathrm{mL})$.

Biochemical analysis of conidial cell surface hydrophobicity (CSH) in A. fumigatus. Hydrophobicity of the conidial suspension was assayed by two-phase partitioning with hexadecane as the hydrocarbon phase (Pihet et al. 2009). Control (untreated) as well as isoeugenol treated $A$. fumigatus conidia were harvested in 1 xPBS and absorbance was set to 0.30 at $630 \mathrm{~nm}$. Hexadecane $(500 \mu \mathrm{L})$ was added to conidial suspension, vortexed ( $2 \mathrm{~min}$ ) at an interval of 30 s and incubated for $10 \mathrm{~min}$ at room temperature for 
phase separation. The absorbance of the aqueous phase was measured at $630 \mathrm{~nm}$ and compared to the initial absorbance.

Percentage reduction in cell surface hydrophobicity (\%CSH) was calculated for treated as well as untreated $A$. fumigatus conidia using the formula:

$$
\% \mathrm{CSH}=\frac{\mathrm{A} 1-\mathrm{A} 2}{\mathrm{~A} 1} \times 100
$$

where A1 is absorption before addition of hydrocarbon; $\mathrm{A} 2$ is absorption after addition of hydrocarbon. Another assay conducted to check the conidial surface hydrophobicity was droplet deposition test. Treated and untreated fungal mycelia along with conidia were placed on the clean glass slide. Approximately, $10 \mu \mathrm{L}$ of sterile water was placed on it for $5 \mathrm{~min}$. The photograph was captured after 5 min following the deposition of droplets (Chau et al. 2010).

Extraction of conidial melanin. The extraction of conidial melanin from treated and control (untreated) $A$. fumigatus was performed as described by Kumar et al. (2011) with modifications. From 4 days grown cultures, conidia were harvested in $1 \times$ PBS by centrifugation (7000 $\mathrm{g}, 5 \mathrm{~min}$ ). The pellet was washed twice with distilled water. For melanin extraction, pellet of $A$. fumigatus conidia was autoclaved by resuspending in $3 \mathrm{~mL}$ of $1 \mathrm{M} \mathrm{KOH}$. Further, acid hydrolysis was done to purify the extracted melanin by adding $5 \mathrm{~mL}$ of $7 \mathrm{M} \mathrm{HCl}$ in a sealed glass vial for $2 \mathrm{~h}$ at $100^{\circ} \mathrm{C}$. After cooling, precipitate was recovered by centrifugation ( $5000 \mathrm{~g} ; 10 \mathrm{~min}$ ). The precipitate was suspended in $100 \mathrm{mM}$ borate buffer and UV-visible absorption spectrum was recorded in the wavelength range $(220-800 \mathrm{~nm})$ on a UV-visible spectrophotometer. $100 \mathrm{mM}$ borate buffer was used as a blank. The experiment was conducted in triplicates.

Analysis of A. fumigatus biofilm formation. The biofilm of $A$. fumigatus was cultured in a 12-well polystyrene plate at calculated IC $\mathrm{C}_{50}$ of isoeugenol (Manavathu et al. 2014). To visualise the effect of isoeugenol scanning electron microscopy (SEM) was conducted and samples were processed as described by González-Ramírez et al (2016), with minor modifications. The A. fumigatus biofilm topology of isoeugenol treated and control (untreated) were viewed under SEM (Zeiss SEM, MA EVO -18 Special Edition).

Scanning and transmission electron microscopy of treated A. fumigatus conidia. Isoeugenol treated conidial surfaces of $A$. fumigatus were also analysed using SEM. The conidia were harvested, washed and fixed in $4 \%$ glutaraldehyde in 1 xPBS under vacuum for $24 \mathrm{~h}$. After washing, the cells were post-fixed with $1 \%$ osmium tetroxide for $60 \mathrm{~min}$ and dehydrated by passage through ethanol solutions of increasing concentration. The samples were then mounted on aluminium sheet and coated with gold-palladium alloy. The observations were made using a Zeiss SEM, MA EVO -18 Special Edition (Pihet et al. 2009).

For TEM analysis, conidia were harvested, washed and fixed overnight at room temperature with $2.5 \%$ glutaraldehyde in $0.1 \mathrm{M}$ sodium cacodylate buffer $(\mathrm{pH} 7.4)$. Conidia were incubated for $1.5 \mathrm{~h}$ at $20^{\circ} \mathrm{C}$ in a 
solution of $4 \%$ formaldehyde $-1 \%$ glutaraldehyde in $0.1 \%$ PBS and then incubated in $2 \%$ osmium tetraoxide for $1.5 \mathrm{~h}$. Dehydration was accomplished by serial washings in graded ethanol solutions of $50-$ $95 \%$ for $10 \mathrm{~min}$, followed by two final washes in $100 \%$ ethanol for $15 \mathrm{~min}$. The cells were embedded in Spurr's resin, sectioned onto nickel grids and examined on a JEOL 2100F transmission electron microscope to obtain micrographs (Graham and Orenstein 2007).

\section{Gene expression analysis.}

Primer designing: The conidial hydrophobin RodA gene, transcriptional regulator genes MedA, SomA, polyketide synthase gene $p k s P$ and tubulin (Tub) CDS gene sequences were downloaded from NCBI (https://www.ncbi.nlm.nih.gov/pubmed) database for designing the primer for expression analysis. The primers were designed by Primer 3 software (http://primer3.ut.ee/) (Untergasser et al. 2012). The primer sequences were then analyzed for potential hair pin formation and self-complementarity (http://www.basic.northwestern.edu/biotools/oligocalc.html). The details of primers are given in Table 1.

Table 1 Gene specific primers used for qRT-PCR.

\begin{tabular}{|lllll|}
\hline S. No. & Gene name & Gene reference ID & Primer sequence (5'-3') & Amplicon size (bp) \\
\hline 1. & RodA & U06121.1 & F: TTGCATACCACATTCCACTC & 186bp \\
& & & R: CTTGTCAACGAGAGTGATGG & \\
\hline 2. & MedA & XM_750565.1 & F: GCCTTGCTAGGTAAGTTTGT & 348bp \\
& & & R: TGTTCCTTCTGAACCTCTC & \\
\hline 3. & SomA & XM_741613.1 & F: ACGTGGCTATCATGAATGTG & 300bp \\
& & & R: TACTGTCTCACGTCGTTGCT & \\
\hline 4. & NksP & NC_007195.1 & F-GTCTACCTTCCTCACGACC & 167bp \\
& & & R-CAGCGTAGAGAGACGATGG & \\
\hline 5. & Tub & NC_007194.1 & F-TTGACCCAGCAGATGTTCG & 174bp \\
& & & R-GGGAATCCACTCAACGAAG & \\
\hline
\end{tabular}

Expression profiling of genes by qRT-PCR: Expression of the genes of interest was quantified by qRT-PCR analysis as described previously (Gupta et al. 2019). Two micrograms of total RNA of each sample (isoeugenol treated and $A$. fumigatus control) were used to synthesize first-strand cDNA by oligo (dT)-18 primer using the Hi- cDNA synthesis Kit (HiMedia). Real-time PCR was performed using an ABI QuantStudio 3 (Applied Biosystems, Streetsville, Canada), and amplification products were detected with SYBR-green master mix (HiMedia) for fungal gene expression.

The relative quantification of individual gene expression was performed using the comparative threshold cycle method. The amplification program used for real time was $95^{\circ} \mathrm{C}$ for $3 \mathrm{~min}, 40$ cycles at $95^{\circ} \mathrm{C}$ for $30 \mathrm{~s}$, 
$60^{\circ} \mathrm{C}$ for $30 \mathrm{~s}, 72^{\circ} \mathrm{C}$ for $30 \mathrm{~s}$. The melt curve analysis was at $95^{\circ} \mathrm{C}$ for $15 \mathrm{~s}, 60^{\circ} \mathrm{C}$ for $60 \mathrm{~s}, 72^{\circ} \mathrm{C}$ for $30 \mathrm{~s}$ and holding stage $10 \mathrm{~s}$. Fungal gene expression was normalized to $A$. fumigatus tubulin expression, and relative expression was estimated using the formula $2^{-\Delta \Delta C \mathrm{Ct}}$, where

$$
\Delta \Delta C t=\frac{[(\mathrm{Ct} \text { target gene }) \text { sample }-(\mathrm{Ct} T u b) \text { sample }]}{[(\text { Ct target gene }) \text { reference }-(\mathrm{Ct} T u b) \text { reference }]}
$$

The results were analyzed using ABI QuantStudio 3 software and genes were considered differentially expressed, if they were at least two-fold up- or down-regulated.

In-silico screening of isoeugenol for therapeutic activity. The qualitative measurements of absorption, distribution, metabolism, excretion, and toxicity of bioactive compound isoeugenol were predicted by using SwissADME program (http://www.swissadme.ch/index.php) (Sharma et al. 2020). The major ADME-Tox properties include molecular weight, $\mathrm{H}$-bond acceptor, $\mathrm{H}$-bond donors, predicted octanol/water partition coefficient (MLogP), total polar surface area (TPSA), Lipinski (drug likeness), Gastrointestinal (GI) absorption, Blood brain barrier (BBB) permeant and bioavailability score. The parameters deployed to predict the physicochemical properties of the compound and describe its disposition within the host organism are summarized in Table 2 (Lipinski et al. 2001).

Molecular docking. The protein structure of RodA hydrophobin from A. fumigatus (PDB ID: 6GCJ) was downloaded from RCSB protein databank (http://www.rcsb.org). Initially, different tasks were performed for protein preparation such as charge assignment, solvation parameters and fragmental volumes using SPDVB-4.10 version (Morris et al. 2009). The protein molecules were further optimized using AutoDock4 version 4.2.6 tool for molecular docking (Morris et al. 2009). The three-dimensional (3D) structure of isoeugenol was downloaded from Drugbank database.

Using AutoDock4 tool, ligand isoeugenol was docked at the bioactive catalytic site of the targeted protein RodA hydrophobin. For protein-ligand interactions, the Lamarckian genetic algorithm was utilized to perform the docking with the pre-set parameters. The total number of poses was set to 50 . Poses were further clustered using all atoms RMSD cut off value of $0.3 \AA$ to remove redundancy and on an average 20 cluster representatives were kept. All other parameters for docking and scoring were used as default sets. The protein structure was kept rigid in all steps. To visualise the protein-ligand interactions, Discovery Studio Visualizer (v20.1.0.19295) was used (http://accelrys.com/products/collaborativescience/biovia-discovery-studio/visualization-download.php).

Statistical analysis. For the statistical analysis, unpaired t-test was used to compare the results of CSH assay and gene expression analysis for isoeugenol treated culture with wild type A. fumigatus. All experiments were conducted in biological triplicates. All the statistics was performed using GraphPad Prism software 8.0.2.263 version and Microsoft Excel. $P<0.05$ was considered statistically significant.

\section{Results}


Minimum inhibitory concentration (MIC) of isoeugenol. MIC and $\mathrm{IC}_{50}$ of bioactive compound isoeugenol were calculated as $312 \mu \mathrm{g} / \mathrm{mL}$ and $156 \mu \mathrm{g} / \mathrm{mL}$, respectively against $A$. fumigatus. Further, all biochemical and molecular assays of $A$. fumigatus were conducted at calculated $\mathrm{IC}_{50}$ value of isoeugenol. Positive and solvent control well showed characteristic greenish-grey $A$. fumigatus conidia whereas isoeugenol treated well at $\mathrm{IC}_{50}$ depicted white pigment-less conidia.

Biochemical analysis of conidial cell surface hydrophobicity (CSH) in A. fumigatus. For fungal colonization and pathogenesis, adhesion of pathogen on the host surface is a crucial step. In $A$. fumigatus, a statistical decrease in the biochemical CSH value was observed in isoeugenol treated culture as compared to the control (untreated). The calculated reduction in $\mathrm{CSH}$ percentage was $72.46 \%$ in isoeugenol treated $A$. fumigatus conidia. (Fig. $1 \mathrm{~A} ; \mathrm{p}<0.005$ ). In addition, hydrophobicity of conidial surface was visualised by dropping sterile water onto the fungal surface. As water droplets were placed onto the fungal surface, it immediately soaked by isoeugenol treated culture. However, untreated dark pigmented conidia of $A$. fumigatus unable to absorb water due to its hydrophobic characteristic (Fig. 1B).

Extraction of conidial melanin. The absorption spectra showed characteristic absorption peaks in the UV region ranging from $250-290 \mathrm{~nm}$ in isoeugenol treated and untreated $A$. fumigatus, but not in the visible region. The overall characteristic absorption peak was shown at $270 \mathrm{~nm}$ (Fig. S1). In control and treated samples, optical density at $270 \mathrm{~nm}$ was 1.1 and 0.42 respectively. The spectra showed a significant reduction in melanin formation in isoeugenol treated conidia as compared to control (untreated).

Scanning electron microscopy of treated A. fumigatus conidia and its biofilm. A. fumigatus conidial surface morphology as well as changes in fungal biofilm after isoeugenol treatment was analyzed and compared to wild type control via SEM. Alteration in conidial cell surface morphology was observed at $\mathrm{IC}_{50}$ treated $A$. fumigatus. Isoeugenol treated conidial surface was observed as smooth without any protrusions whereas the wild type conidia showed echinulate surface (Fig. 2A and 2B). In electron micrographs, closely packed $A$. fumigatus conidia were visualized in untreated control, on the other hand, the presence of isoeugenol reduced the hydrophobic interaction among conidia (Fig. 2C and 2D).

Transmission electron microscopy (TEM) analysis confirmed presence of protrusions in control conidia (untreated; Fig. 2E) as observed in Fig. 2A whereas isoeugenol treated conidial section revealed absence of protrusion on outer surface with visible clear inner cell wall surface indicating absence of melanin layer (Fig. 2F).

In SEM micrographs, defected $A$. fumigatus biofilm viewed at $\mathrm{IC}_{50}$ of isoeugenol where only hyphal growth with lack of ECM (Fig. 3B). However, in wild type control highly compact intermingled hyphae covered with high amount of ECM were observed which was the characteristic feature of fungal biofilm (Fig. 3A). Isoeugenol possess anti-biofilm effect on A. fumigatus with absence of ECM; also, able to reduce the adherence properties, responsible to initiate the fungal infection. 
Gene expression analysis of hydrophobin gene, RodA. The impact of isoeugenol treatment on rodlet gene $\operatorname{Rod} A$, transcription regulatory genes of $A$. fumigatus MedA, SomA and $p k s P$ was investigated by reverse transcription followed by qRT-PCR for differential gene expression. Isoeugenol treatment $(0.156 \mathrm{mg} / \mathrm{mL})$ led to a significant down-regulation of RodA gene as well as transcriptional regulators MedA, SomA and $p k s P$ gene transcripts in comparison with control (untreated) (Fig. 4). The complete expression data was normalized by housekeeping gene $\beta$-tubulin. Gene expression data expressed as $2^{-\Delta \Delta C t}$ is the mean of at least 3 replicates \pm standard error.

In-silico screening of isoeugenol for therapeutic activity. Isoeugenol was studied for its physicochemical properties and ADME to screen bioavailability via oral route. Table 2 shows all five key criteria required for any compound to possess drug-likeness properties. ADME analysis suggested that the isoeugenol has potential therapeutic abilities in reference to Lipinski's rule of five: molecular weight less than $500 \mathrm{~g} / \mathrm{mol}$, hydrogen bond donor less than 5 , hydrogen bond acceptor less than 10 and lipophilicity (Log P) value less than 5. Drug likeness properties are in favour as it is following Lipinski's, verber, egan rule having bioavailability score of 0.55 with high $\mathrm{GI}$ absorption and BBB permeant.

The isoeugenol bioavailability radar (Fig. 5) revealed that coloured zone is a suitable physicochemical space for oral bioavailability. The parameters taken into consideration for analysis were flexibility (FLEX), lipophilicity (LIPO), saturation (INSATU), size (SIZE), polarity (POLAR) and solubility (INSOLU). All the mentioned parameters were in coloured zone except INSATU which was 0.20 .

Table 2

In-silico prediction of drug-likeness of isoeugenol via SwissADME.

\begin{tabular}{|llll|}
\hline S. No. & Property & Criterion & in-silico analysis of Isoeugenol \\
\hline 1. & Molecular weight & $<500 \mathrm{~g} / \mathrm{mol}$ & $164.20 \mathrm{~g} / \mathrm{mol}$ \\
\hline 2. & Hydrogen donor & $<6.0$ & 01 \\
\hline 3. & Hydrogen acceptor & $<5.0$ & 02 \\
\hline 4. & Rotational bonds & $<10.0$ & 02 \\
\hline 5 & Topological Polar Surface Area (TPSA) & $<140 \AA^{2}$ & $29.46 \AA^{2}$ \\
\hline 6. & Log P (lipophilicity) & $<5$ & 2.38 \\
\hline 7. & Log S (Solubility) & - & -3.01 \\
\hline 8. & Bioavailability Score & - & 0.55 \\
\hline & Lipinski Violations & Nil \\
\hline
\end{tabular}

Molecular docking. Molecular docking was performed to evaluate the interaction of isoeugenol with RodA hydrophobin from A. fumigatus. Hydrophobin protein encoded by RodA gene was targeted and the docking pocket was formed at the catalytic sites of the respective protein. The docking pocket developed 
around the amino acid Cys-64 having coordinates $(1.321,-6.533,2.078)$ in the chain $\mathrm{A}$. Besides, the binding affinity of interacted compounds at the active site was assessed. The isoeugenol exhibited the docked score of $-4.54 \mathrm{Kcal} / \mathrm{mol}$ and formed four hydrogen bonds with amino acid residues Lys-126, Cys127, GIn-129 and Lys-128. It also formed a pi-pi bond with Cys-64 and Cys-127 amino acid in the catalytic domain (Fig. 6).

\section{Discussion}

Several different features of $A$. fumigatus physiology have been proposed that accounts for its high virulence, including conidial size, their ubiquitous abundance and hydrophobicity of the cell wall (Abad et al. 2010; van de Veerdonk et al. 2017). The surface of $A$. fumigatus conidia is masked by a layer of hydrophobin proteins (Linder et al. 2005; Aimanianda et al. 2009) which forms rodlets on the surface of A. fumigatus conidia (Paris et al. 2003; Valsecchi et al. 2019). The rodlet layer is amphipathic and it renders the conidial surface resistant to wettability as well as facilitates easy spore dispersion. It consists of rodlet proteins RodA and RodB wherein only RodA contributes majorly towards conidial hydrophobicity, immune inertness against macrophages (Paris et al. 2003) and also restricts the entry of hydrophilic molecules like oxidant components of phagocytes (Rementeria et al. 2005). Along with hydrophobins, fungal adhesion to biotic and abiotic surfaces signifies initial stage towards establishment of fungal biofilms (Oshiro et al. 2019). According to the literature, biofilm is an important structure for fungal virulence and provide protection from host response as well as antifungal drugs. Hydrophobins do not play any significant role in biofilm formation and its adherence (Valsecchi et al. 2017). There are reported regulatory genes underlying biofilm formation that are SomA and MedA in A. fumigatus-mediated invasive diseases that has now been studied in detail (Gravelat et al. 2010; Zhang et al. 2018; Chen et al. 2020).

Various studies have reported that phenolic compounds are mainly responsible for the antimicrobial properties of plant essential oils (Gutiérrez-Larraínzar et al. 2012; Zabka and Pavela 2013; Medeiros et al. 2020). Antibacterial (gram positive/gram negative) and antifungal (Candida spp.) action of isoeugenol (isomer of phenolic compound eugenol) has been previously evaluated, suggesting that it targets cell membrane by triggering intracellular acidification and cell membrane breakage (Bhatia et al. 2012; Hyldgaard et al. 2015). The presence of free hydroxyl (-OH) from the aromatic chain of isoeugenol is one of the structural parameters involved in the antimicrobial activity of this phytoconstituent, however, molecular properties such as hydrophobicity, refractivity and molecular geometry also seem to be related to the inhibitory capacity of isoeugenol on Candida spp. (Dambolena et al. 2012; Siddiqui et al. 2013). It has been reported that it is an effective molecule in controlling the establishment and progression of candidiasis by inhibiting the formation of virulence structures (chlamidoconidia, blastoconidia and pseudo-hyphae) in Candida albicans (Mayer et al. 2013; Medeiros et al. 2020). Thus, the present study explored the effects of phenolic compound isoeugenol on conidial hydrophobicity and biofilm formation of $A$. fumigatus. 
We investigated the antifungal action of isoeugenol on filamentous fungi $A$. fumigatus. The minimum inhibition concentration of isoeugenol was observed at $0.312 \mathrm{mg} / \mathrm{mL}$ and $\mathrm{IC}_{50}$ at $0.156 \mathrm{mg} / \mathrm{mL}$. A study conducted on filamentous fungi Penicillium citrinum reported the MIC of isoeugenol in the range of 0.256- $0.032 \mathrm{mg} / \mathrm{mL}$ (Ferreira et al. 2018) whereas antibacterial effect in range 0.6-1.0 mg/mL (Hyldgaard et al. 2015). Pizzolitto et al. (2015) reported that isoeugenol possesses higher antifungal activity as compared to eugenol and other phenolic compounds. Thus, isoeugenol is a promising candidate which can be further evaluated as a potential antifungal molecule with its molecular target approach.

In presence of isoeugenol, conidial cell surface hydrophobicity of $A$. fumigatus was analysed which showed a significant decrease with increased wettability of the conidia. Similar results on hydrophobicity have also been reported in A fumigatus mutant by Pihet et al (2009) and Girardin et al (1999). Isoeugenol treated $A$. fumigatus produced pigment-less conidia whereas wild-type control was of greyish green colour. In addition, the UV-vis spectrum showed that the DHN-melanin was reduced in treated conidia due to its inability to produce conidial pigment. Various other studies have also suggested that the absence of rodlet layer and melanin pigment corresponds to altered hydrophobicity of the $A$. fumigatus conidia (Aimanianda et al. 2009; Zhang et al. 2011; Hoda et al. 2020). The white pigment-less, smooth walled conidia of $A$. fumigatus have been reported in $\triangle p k s P$ (DHN-melanin biosynthesis pathway mutant) (Bernard and Latgé 2001) as well as rodlet mutant (Paris et al. 2003). Subsequently, scanning electron micrographs suggested an alteration in the spore cell wall with loss of protrusion in isoeugenol treated conidia whereas wild type conidia showed a distinct ornamentation on their surface. At low magnification, closely packed conidia as seen in control (untreated) sample, were not observed in isoeugenol treated $A$. fumigatus. The conidial samples were later subjected to TEM observations where treated conidia revealed marked modifications in the outer membrane with loss of protrusions and lack of melanin content in comparison to wild type control. On other hand Fig. 4 depicted that presence of isoeugenol inhibited the ECM formation, which in turn decreases the compact hyphal structure of $A$. fumigatus biofilm. These micrographs suggested that isoeugenol plays a major role in inhibition of outer cell wall components via interfering their biosynthesis and its biofilm formation. In addition, the altered conidial surface could enhance drug sensitivity of $A$. fumigatus. Aimanianda et al (2009) reported that altered conidial surface also induces inflammatory cytokines, chemokines and reactive oxygen intermediates to activate host immune response.

Conidial surface study was coupled with the analysis of expression levels of hydrophobicity- related gene RodA, transcription regulators MedA, SomA and DHN-melanin biosynthesis gene pksP in isoeugenol treated as well as wild type $A$. fumigatus. Compared to wild type, the expression levels of studied genes (RodA, MedA SomA and pksP) were significantly downregulated at $I_{50}$ treated $A$. fumigatus (Fig. 5). The gene expression of RodA gene responsible for rodlet formation and transcriptional regulator MedA and SomA governs adherence, conidiation, host interaction and biofilm formation in $A$. fumigatus (Gravelat et al. 2010; Lin et al. 2015; Valsecchi et al. 2019). Moreover, in mutant $\triangle R o d A$ conidia, underneath layer gets exposed which is composed of glycoproteins, chitin and $\beta-(1,3)$ - glucan (Valsecchi et al. 2020). These

Page $11 / 23$ 
components are pathogen associated molecule patterns and their exposure on the conidial surface actively induces the host immune response. Deletion of rodA gene impacts drug sensitivity of the fungi (Valsecchi et al. 2019). Another study on $\triangle M e d A$ mutant which analysed the role of gene MedA in the adherence of $A$. fumigatus to epithelial cells $A 549$ as well as in biofilm formation (Gravelat et al. 2010) reported that $\triangle M e d A$ mutant strain was unable to induce any epithelial cell damage with decreased adherence, and stimulation of cytokine production in comparison to the wild type. It is still unclear whether MedA directly interacts with substrates to mediate adherence or not. Probably, MedA serves as a regulatory protein controlling the expression of downstream adhesion genes (Gravelat et al. 2010). The $\triangle M e d A$ and $\triangle p k s P$ mutant conidia are also reported to undergo de-pigmented morphology and has been linked to A. fumigatus virulence (Gravelat et al. 2010; Hoda et al. 2020). SomA gene expression was highly downregulated in our study, these results corroborated by scanning electron micrographs depicted loss of ECM and biofilm formation in presence of isoeugenol. There are certain strong evidences suggesting downregulation of SomA gene and its effects on $A$. fumigatus biofilm formation and enhances sensitivity to cell wall stressors (Lin et al. 2015; Zhang et al. 2018; Chen et al. 2020). SomA governs GAG biosynthetic genes as well as cell wall related genes under normal and cell wall stress conditions.

Pharmacokinetic properties of isoeugenol were studied via in-silico analysis. SwissADME web tool was used to predict the physiochemical properties and drug likeness of molecule to further analyse the molecule on other parameters. It takes into account six important physicochemical properties including flexibility, lipophilicity, saturation, size, polarity and solubility (Pires et al. 2015). In the present study, drug likeness properties of isoeugenol were in favour as it follows Lipinski's, verber, egan rule. The pink area of radar plot showed that isoeugenol satisfies all the mentioned properties except saturation which should be less than 0.25 (http://www.swissadme.ch/index.php). Lipinski's rule of five states molecular weight less than 500 , hydrogen bond acceptor less than 10, hydrogen bond donor less than 5 and logP value should be less than 5 . On other hand, verber rule states rotatable bonds should be less than 10 and polar surface area should not be more than $140 \AA$ are orally active (Cheng et al. 2012). Isoeugenol's computational ADME studies suggested that it can be considered as a drug-like molecule and similar results were reported by Ferreira et al (2018).

In present study, molecular docking was conducted at $A$. fumigatus RodA protein catalytic site that consists of Cys 64 as one of the amino acids. The docking score obtained between ligand isoeugenol and cysteine residue active site of RodA protein was $-4.54 \mathrm{Kcal} / \mathrm{mol}$ with four hydrogen bonds formation. The secondary structure of RodA protein consists of a central $\beta$-barrel composed of two curved antiparallel $\beta$-sheets, two relatively long a-helices and two short $3_{10}$ helices (Valsecchi et al. 2019). The structure of RodA protein comprises four-disulfide bridges formed between eight cysteine residues and two amyloidogenic regions. According to Valsecchi et al. (2020), any point mutations on this conserved region may lead to alteration in the cell wall organisation rendering conidial surface hydrophilic. And, out of eight cysteine residues isoeugenol interacted with four cysteine residues: two formed hydrogen bonds and two formed van der waal interaction. The counting of these intermolecular interactions formed 
between receptor and ligand is calculated in terms of scoring function (Oda et al. 2006). Consequently, it is very important to understand molecular mechanisms that are involved in reduction of conidial hydrophobicity, biofilm formation in presence of isoeugenol as this work will provide new approaches to combat the infection caused by $A$. fumigatus.

\section{Conclusion}

The present study concluded that isoeugenol is capable of inhibiting hydrophobin formation on $A$. fumigatus conidia, which is one of the crucial factors for adherence as well as initiation of infection in the host cell. The compound also prevents its biofilm formation. It downregulated the expression of RodA gene responsible for rodlet formation and transcriptional regulators MedA and SomA that regulates downstream genes responsible for adherence, virulence and biofilm formation in $A$. fumigatus. the compound also inhibits gene expression which is first gene responsible for DHN-melanin pigmentation in A. fumigatus.

\section{Declarations}

\section{Acknowledgements}

The authors would like to thank AIRF- JNU, New Delhi, India for performing transmission electron microscopy. The authors are grateful to the Amity University, NOIDA for providing the facilities to carry out the research work.

\section{Funding}

The authors would like to thank Department of Science and Technology-Science and Engineering Research Board (DST-SERB) (EMR/2016/005752), New Delhi, Govt. of India for their financial support. The authors thank Amity University Uttar Pradesh, India, for providing infrastructure for research.

\section{Author's contributions}

LG performed literature search, experimental part and the manuscript drafting; PS also performed experiments and helped in manuscript editing; AKB critically reviewed and corrected the manuscript; and PV conceptualised the idea as well as critically analysed the results and manuscript.

\section{Conflicts of interest}

No potential conflict of interest was reported by the authors. The authors are responsible for the content and paper writing.

\section{Compliance with ethical standards}

Ethics approval- Not applicable 
Consent to participate- Not applicable

Consent for publication- Not applicable

Availability of data and materials- Not applicable

\section{References}

Abad A, Fernández-Molina JV, Bikandi J, et al (2010) What makes Aspergillus fumigatus a successful pathogen? Genes and molecules involved in invasive aspergillosis. Rev Iberoam Micol 27:155-182. https://doi.org/10.1016/j.riam.2010.10.003

Abastabar M, Hosseini T, Valadan R, et al (2019) Novel Point Mutations in cyp51A and cyp51B Genes Associated with Itraconazole and Posaconazole Resistance in Aspergillus clavatus Isolates. Microb Drug Resist 25:652-662. https://doi.org/10.1089/mdr.2018.0300

Abdallah QA, Choe S-I, Campoli P, et al (2012) Conserved C-Terminal Domain of the Aspergillus fumigatus Developmental Regulator MedA Is Required for Nuclear Localization, Adhesion and Virulence. PLOS ONE 7:e49959. https://doi.org/10.1371/journal.pone.0049959

Aimanianda V, Bayry J, Bozza S, et al (2009) Surface hydrophobin prevents immune recognition of airborne fungal spores. Nature 460:1117-1121. https://doi.org/10.1038/nature08264

Aldholmi M, Marchand P, Ourliac-Garnier I, et al (2019) A Decade of Antifungal Leads from Natural Products: 2010-2019. Pharmaceuticals 12:182. https://doi.org/10.3390/ph12040182

Alexander BD, Clinical and Laboratory Standards Institute (2017) Reference method for broth dilution antifungal susceptibility testing of filamentous fungi

Bayry J, Beaussart A, Dufrêne YF, et al (2014) Surface structure characterization of Aspergillus fumigatus conidia mutated in the melanin synthesis pathway and their human cellular immune response. Infect Immun 82:3141-3153. https://doi.org/10.1128/IAI.01726-14

Bernard M, Latgé JP (2001) Aspergillus fumigatus cell wall: composition and biosynthesis. Med Mycol 39 Suppl 1:9-17

Bhatia R, Shreaz S, Khan N, et al (2012) Proton pumping ATPase mediated fungicidal activity of two essential oil components. J Basic Microbiol 52:504-512. https://doi.org/10.1002/jobm.201100272

Borman AM, Palmer MD, Fraser M, et al (2020) COVID-19-Associated Invasive Aspergillosis: Data from the UK National Mycology Reference Laboratory. J Clin Microbiol 59:JCM.02136-20, e02136-20. https://doi.org/10.1128/JCM.02136-20 
Brauer VS, Rezende CP, Pessoni AM, et al (2019) Antifungal Agents in Agriculture: Friends and Foes of Public Health. Biomolecules 9:E521. https://doi.org/10.3390/biom9100521

Campaniello D, Corbo MR, Sinigaglia M (2010) Antifungal Activity of Eugenol against Penicillium, Aspergillus, and Fusarium Species. J Food Prot 73:1124-1128. https://doi.org/10.4315/0362-028x73.6.1124

Cerqueira GC, Arnaud MB, Inglis DO, et al (2014) The Aspergillus Genome Database: multispecies curation and incorporation of RNA-Seq data to improve structural gene annotations. Nucleic Acids Res 42:D705-710. https://doi.org/10.1093/nar/gkt1029

Chau HW, Goh YK, Si BC, Vujanovic V (2010) Assessment of alcohol percentage test for fungal surface hydrophobicity measurement. Letters in Applied Microbiology 50:295-300.

https://doi.org/10.1111/j.1472-765X.2009.02791.x

Chen Y, Le Mauff F, Wang Y, et al The Transcription Factor SomA Synchronously Regulates Biofilm Formation and Cell Wall Homeostasis in Aspergillus fumigatus. mBio 11:e02329-20.

https://doi.org/10.1128/mBio.02329-20

Cheng F, Li W, Zhou Y, et al (2012) admetSAR: a comprehensive source and free tool for assessment of chemical ADMET properties. J Chem Inf Model 52:3099-3105. https://doi.org/10.1021/ci300367a

Croft CA, Culibrk L, Moore MM, Tebbutt SJ (2016) Interactions of Aspergillus fumigatus Conidia with Airway Epithelial Cells: A Critical Review. Front Microbiol 7:472.

https://doi.org/10.3389/fmicb.2016.00472

Dagenais TRT, Giles SS, Aimanianda V, et al (2010) Aspergillus fumigatus LaeA-Mediated Phagocytosis Is Associated with a Decreased Hydrophobin Layer. Infect Immun 78:823-829.

https://doi.org/10.1128/IAl.00980-09

Dambolena JS, López AG, Meriles JM, et al (2012) Inhibitory effect of 10 natural phenolic compounds on Fusarium verticillioides. A structure-property-activity relationship study. Food Control 28:163-170. https://doi.org/10.1016/j.foodcont.2012.05.008

Escobar N, Ordonez SR, Wösten HAB, et al (2016) Hide, Keep Quiet, and Keep Low: Properties That Make Aspergillus fumigatus a Successful Lung Pathogen. Front Microbiol 7:438.

https://doi.org/10.3389/fmicb.2016.00438

Ferreira SB, Dantas TB, de Figuerêdo Silva D, et al (2018) In Silico and In Vitro Investigation of the Antifungal Activity of Isoeugenol against Penicillium citrinum. Curr Top Med Chem 18:2186-2196. https://doi.org/10.2174/1568026619666181130141818

Girardin H, Paris S, Rault J, et al (1999) The role of the rodlet structure on the physicochemical properties of Aspergillus conidia. Lett Appl Microbiol 29:364-369. https://doi.org/10.1046/j.1472- 
González-Ramírez Al, Ramírez-Granillo A, Medina-Canales MG, et al (2016) Analysis and description of the stages of Aspergillus fumigatus biofilm formation using scanning electron microscopy. BMC Microbiology 16:243. https://doi.org/10.1186/s12866-016-0859-4

Graham L, Orenstein JM (2007) Processing tissue and cells for transmission electron microscopy in diagnostic pathology and research. Nat Protoc 2:2439-2450. https://doi.org/10.1038/nprot.2007.304

Gravelat FN, Ejzykowicz DE, Chiang LY, et al (2010) Aspergillus fumigatus MedA governs adherence, host cell interactions and virulence. Cell Microbiol 12:473-488. https://doi.org/10.1111/j.1462-

5822.2009.01408.x

Gupta L, Hoda S, Vermani M, Vijayaraghavan P (2021) Understanding the fundamental role of virulence determinants to combat Aspergillus fumigatus infections: exploring beyond cell wall. Mycol Progress 20:365-380. https://doi.org/10.1007/s11557-021-01677-w

Gupta, Sharma A, Ahluwalia S, Vijayaraghavan P (2019) Myristica fragrans extract inhibits melanin biosynthesis, hyphal growth and appressorium formation in Magnaporthe oryzae. Curr Research Environ \& Applied Mycology (Journal of Fungal Biology) 9(1): 255-270.

Gutiérrez-Larraínzar M, Rúa J, Caro I, et al (2012) Evaluation of antimicrobial and antioxidant activities of natural phenolic compounds against foodborne pathogens and spoilage bacteria. Food Control 26:555563. https://doi.org/10.1016/j.foodcont.2012.02.025

Hagiwara D, Watanabe A, Kamei K, Goldman GH (2016) Epidemiological and Genomic Landscape of Azole Resistance Mechanisms in Aspergillus Fungi. Front Microbiol 7:1382.

https://doi.org/10.3389/fmicb.2016.01382

Hoda S, Vermani M, Joshi RK, et al (2020) Anti-melanogenic activity of Myristica fragrans extract against Aspergillus fumigatus using phenotypic based screening. BMC Complementary Medicine and Therapies 20:67. https://doi.org/10.1186/s12906-020-2859-z

Hyldgaard M, Mygind T, Piotrowska R, et al (2015) Isoeugenol has a non-disruptive detergent-like mechanism of action. Front Microbiol 6:754. https://doi.org/10.3389/fmicb.2015.00754

Kasprzyk I (2008) Aeromycology-main research fields of interest during the last 25 years. Ann Agric Environ Med 15:1-7.

Khalil AA, Rahman U ur, Khan MR, et al (2017) Essential oil eugenol: sources, extraction techniques and nutraceutical perspectives. RSC Adv 7:32669-32681. https://doi.org/10.1039/C7RA04803C

Kumar CG, Mongolla P, Pombala S, et al (2011) Physicochemical characterization and antioxidant activity of melanin from a novel strain of Aspergillus bridgeri ICTF-201. Lett Appl Microbiol 53:350-358. 
Lin C-J, Sasse C, Gerke J, et al (2015) Transcription Factor SomA Is Required for Adhesion, Development and Virulence of the Human Pathogen Aspergillus fumigatus. PLOS Pathogens 11:e1005205. https://doi.org/10.1371/journal.ppat.1005205

Linder MB, Szilvay GR, Nakari-Setälä T, Penttilä ME (2005) Hydrophobins: the protein-amphiphiles of filamentous fungi. FEMS Microbiol Rev 29:877-896. https://doi.org/10.1016/j.femsre.2005.01.004

Lipinski CA, Lombardo F, Dominy BW, Feeney PJ (2001) Experimental and computational approaches to estimate solubility and permeability in drug discovery and development settings. Adv Drug Deliv Rev 46:3-26. https://doi.org/10.1016/s0169-409x(00)00129-0

Manavathu EK, Vager DL, Vazquez JA (2014) Development and antimicrobial susceptibility studies of in vitro monomicrobial and polymicrobial biofilm models with Aspergillus fumigatus and Pseudomonas aeruginosa. BMC Microbiology 14:53. https://doi.org/10.1186/1471-2180-14-53

Mayer FL, Wilson D, Hube B (2013) Candida albicans pathogenicity mechanisms. Virulence 4:119-128. https://doi.org/10.4161/viru.22913

Medeiros D, Oliveira-Júnior J, Nóbrega J, et al (2020) Isoeugenol and Hybrid Acetamides against Candida albicans Isolated from the Oral Cavity. Pharmaceuticals 13:291. https://doi.org/10.3390/ph13100291

Morris GM, Huey R, Lindstrom W, et al (2009) AutoDock4 and AutoDockTools4: Automated docking with selective receptor flexibility. J Comput Chem 30:2785-2791. https://doi.org/10.1002/jcc.21256

Mota Fernandes C, Dasilva D, Haranahalli K, et al (2021) The Future of Antifungal Drug Therapy: Novel Compounds and Targets. Antimicrobial Agents and Chemotherapy 65:e01719-20. https://doi.org/10.1128/AAC.01719-20

Mutlu-Ingok A, Devecioglu D, Dikmetas DN, et al (2020) Antibacterial, Antifungal, Antimycotoxigenic, and Antioxidant Activities of Essential Oils: An Updated Review. Molecules 25:E4711.

https://doi.org/10.3390/molecules25204711

Oda A, Tsuchida K, Takakura T, et al (2006) Comparison of consensus scoring strategies for evaluating computational models of protein-ligand complexes. J Chem Inf Model 46:380-391.

https://doi.org/10.1021/ci050283k

Oshiro KGN, Rodrigues G, Monges BED, et al (2019) Bioactive Peptides Against Fungal Biofilms. Front Microbiol 0: https://doi.org/10.3389/fmicb.2019.02169

Paris S, Debeaupuis J-P, Crameri R, et al (2003) Conidial Hydrophobins of Aspergillus fumigatus. Appl Environ Microbiol 69:1581-1588. https://doi.org/10.1128/AEM.69.3.1581-1588.2003 
Peixoto LR, Rosalen PL, Ferreira GLS, et al (2017) Antifungal activity, mode of action and anti-biofilm effects of Laurus nobilis Linnaeus essential oil against Candida spp. Arch Oral Biol 73:179-185. https://doi.org/10.1016/j.archoralbio.2016.10.013

Pihet M, Vandeputte P, Tronchin G, et al (2009) Melanin is an essential component for the integrity of the cell wall of Aspergillus fumigatus conidia. BMC Microbiology 9:177. https://doi.org/10.1186/1471-21809-177

Pires DEV, Blundell TL, Ascher DB (2015) pkCSM: Predicting Small-Molecule Pharmacokinetic and Toxicity Properties Using Graph-Based Signatures. J Med Chem 58:4066-4072.

https://doi.org/10.1021/acs.jmedchem.5b00104

Pizzolitto RP, Barberis CL, Dambolena JS, et al (2015) Inhibitory Effect of Natural Phenolic Compounds on Aspergillus parasiticus Growth. Journal of Chemistry 2015:e547925.

https://doi.org/10.1155/2015/547925

Rementeria A, López-Molina N, Ludwig A, et al (2005) Genes and molecules involved in Aspergillus fumigatus virulence. Rev Iberoam Micol 22:1-23. https://doi.org/10.1016/s1130-1406(05)70001-2

Sharma A, Goyal S, Yadav AK, et al (2020) In-silico screening of plant-derived antivirals against main protease, 3CLpro and endoribonuclease, NSP15 proteins of SARS-CoV-2. J Biomol Struct Dyn. 8:1-15. https://doi.org/10.1080/07391102.2020.1808077

Siddiqui ZN, Farooq F, Musthafa TNM, et al (2013) Synthesis, characterization and antimicrobial evaluation of novel halopyrazole derivatives. Journal of Saudi Chemical Society 17:237-243. https://doi.org/10.1016/j.jscs.2011.03.016

Szumilak M, Galdyszynska M, Dominska K, et al (2017) Synthesis, Biological Activity and Preliminary in Silico ADMET Screening of Polyamine Conjugates with Bicyclic Systems. Molecules 22:794. https://doi.org/10.3390/molecules22050794

Untergasser A, Cutcutache I, Koressaar T, et al (2012) Primer3-new capabilities and interfaces. Nucleic Acids Res 40:e115. https://doi.org/10.1093/nar/gks596

Valsecchi I, Dupres V, Stephen-Victor E, et al (2017) Role of Hydrophobins in Aspergillus fumigatus. J Fungi (Basel) 4(1). https://doi.org/10.3390/jof4010002

Valsecchi I, Lai JI, Stephen-Victor E, et al (2019) Assembly and disassembly of Aspergillus fumigatus conidial rodlets. The Cell Surface 5:100023. https://doi.org/10.1016/j.tcsw.2019.100023

Valsecchi I, Stephen-Victor E, Wong SSW, et al (2020) The Role of RodA-Conserved Cysteine Residues in the Aspergillus fumigatus Conidial Surface Organization. J Fungi (Basel) 6:151.

https://doi.org/10.3390/jof6030151

Page $18 / 23$ 
van de Veerdonk FL, Gresnigt MS, Romani L, et al (2017) Aspergillus fumigatus morphology and dynamic host interactions. Nature Reviews Microbiology 15:661-674. https://doi.org/10.1038/nrmicro.2017.90

Voltersen V, Blango MG, Herrmann S, et al (2018) Proteome Analysis Reveals the Conidial Surface Protein CcpA Essential for Virulence of the Pathogenic Fungus Aspergillus fumigatus. mBio 9:e01557-18. https://doi.org/10.1128/mBio.01557-18

Zabka M, Pavela R (2013) Antifungal efficacy of some natural phenolic compounds against significant pathogenic and toxinogenic filamentous fungi. Chemosphere 93:1051-1056.

https://doi.org/10.1016/j.chemosphere.2013.05.076

Zarlaha A, Kourkoumelis N, Stanojkovic TP, Kovala-Demertzi D (2014) Cytotoxic activity of essential oil and extracts of Ocimum Basilicum against human carcinoma cells. Molecular docking study of isoeugenol as a potent cox and lox inhibitor. Dig J Nanomater Biostructures 9(3): 907-917

Zhang S, Chen Y, Ma Z, et al (2018) PtaB, a lim-domain binding protein in Aspergillus fumigatus regulates biofilm formation and conidiation through distinct pathways. Cellular Microbiology 20:e12799. https://doi.org/10.1111/cmi.12799

Zhang S, Xia YX, Kim B, Keyhani NO (2011) Two hydrophobins are involved in fungal spore coat rodlet layer assembly and each play distinct roles in surface interactions, development and pathogenesis in the entomopathogenic fungus, Beauveria bassiana. Mol Microbiol 80:811-826.

https://doi.org/10.1111/j.1365-2958.2011.07613.x

\section{Figures}




\section{Cell surface hydrophobicity reduction}
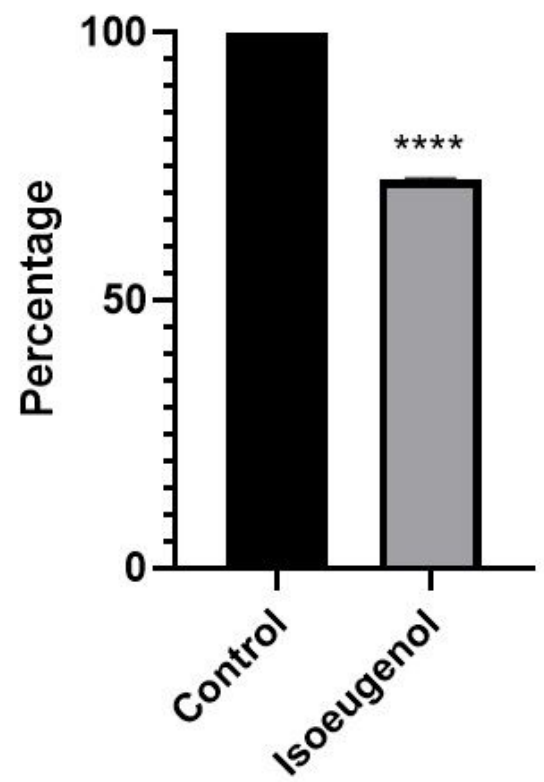

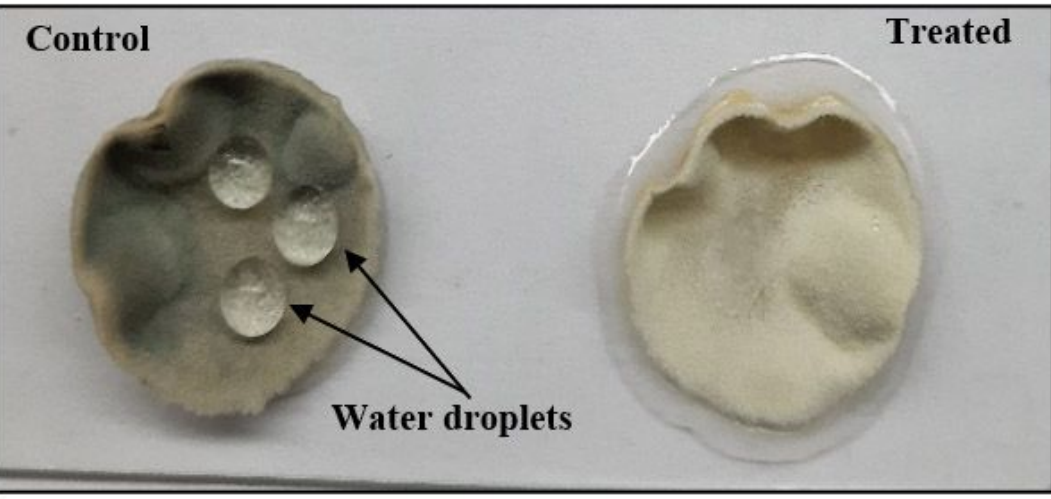

(B)

(A)

\section{Figure 1}

A) Reduction in CSH percentage of A. fumigatus conidia in the presence of isoeugenol. B) $10 \mu \mathrm{L}$ of sterile water was dropped onto the surface of isoeugenol treated and untreated $A$. fumigatus culture. Loss of hydrophobicity is indicated by absorption of the water droplet into the culture
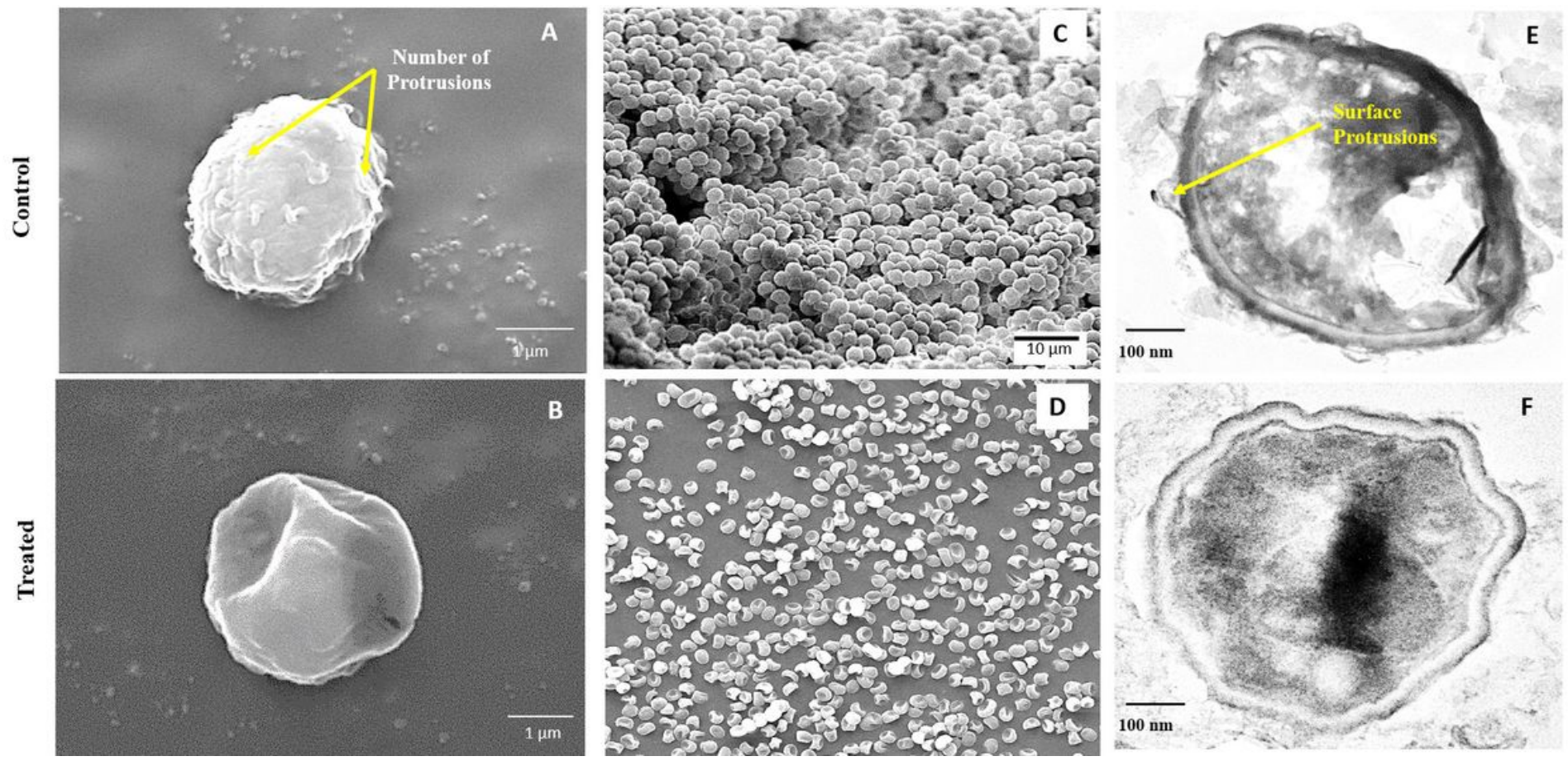

Figure 2 
Visualisation of scanning electron micrographs of $A$. fumigatus conidial surface with $(A)$ presence of protrusions on wild-type conidia, whereas (B) surface morphology with absence of protrusions in isoeugenol treated conidia at magnification of $40 \mathrm{~K} \times(\mathrm{C})$ closely packed hydrophobic wild-type conidia $(D)$ isoeugenol treated $A$. fumigatus conidia at $5 \mathrm{~K} \times$ magnification $(E)$ and $(F)$ loss of protrusions and electron dense melanin layer on A. fumigatus conidial cell wall in comparison to control (untreated) under transmission electron microscope
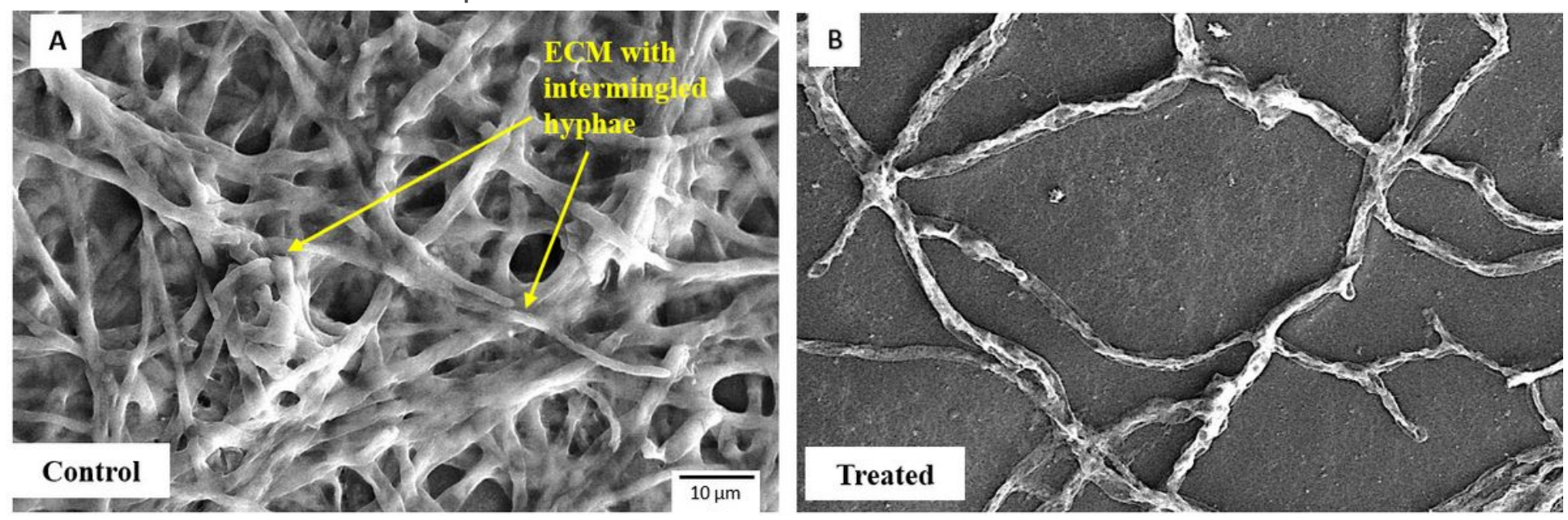

\section{Figure 3}

Electron micrographs of $A$. fumigatus biofilm morphology (A) wild type control (without treatment); (B) isoeugenol treatment with lack of ECM and reduced hyphae at $2 \mathrm{~K} \times$ magnification
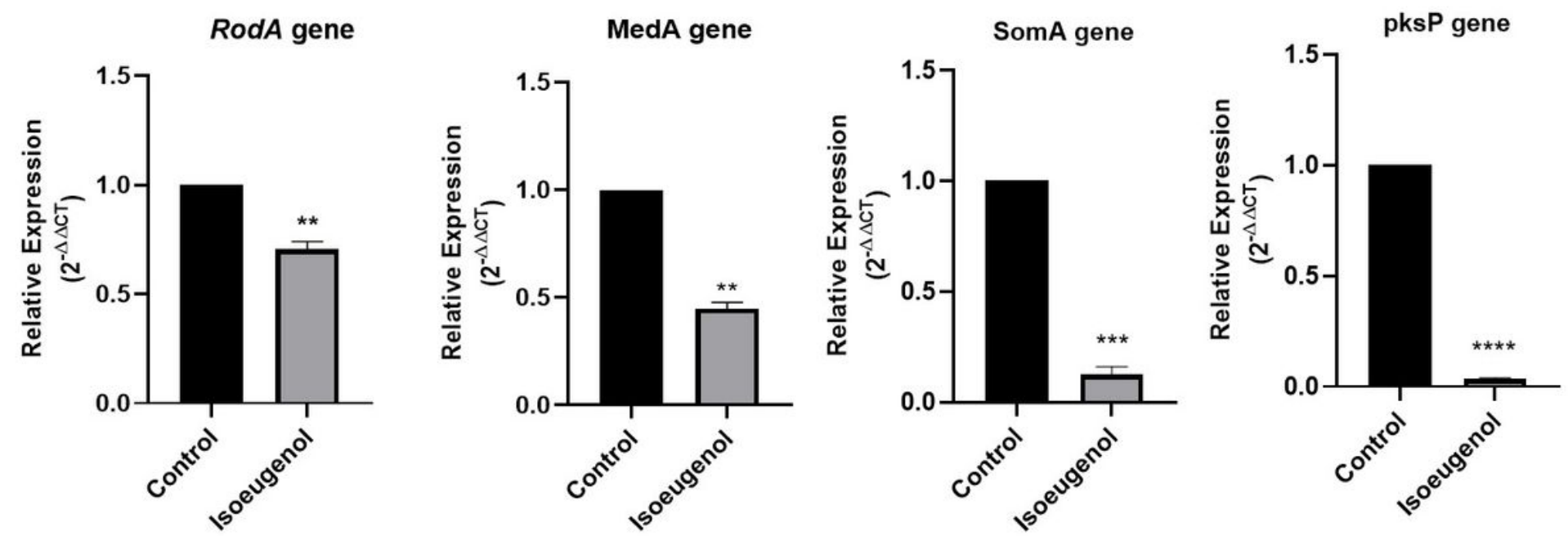

\section{Figure 4}

Relative quantification of RodA, MedA SomA and pksP gene expression (normalised to house-keeping gene $\beta$-tubulin) in A. fumigatus treated with IC50 of isoeugenol. Data reported as mean of fold changes with standard deviation from three independent experiments amplified in triplicates. $p \leq 0.05$ was considered statistically significant 


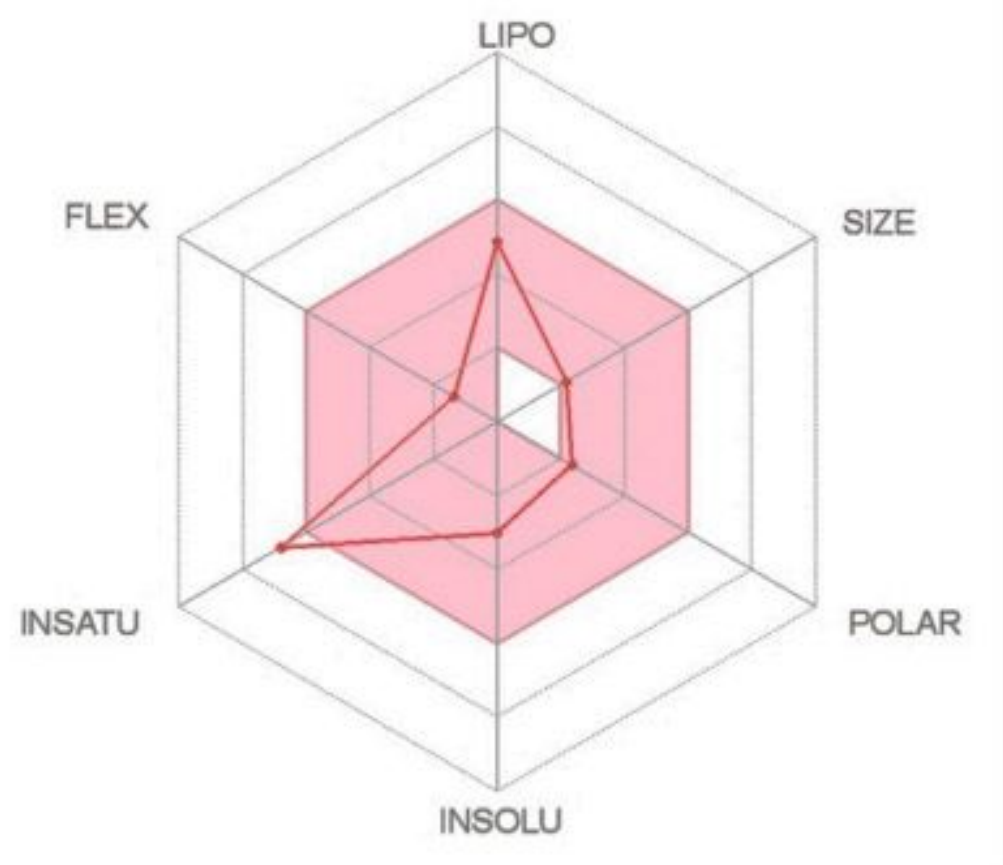

Figure 5

The oral bioavailability radar of isoeugenol using SwissADME predictor. The six physiochemical properties taken into account to consider a molecule as drug-like are FLEX, LIPO, INSATU, SIZE, POLAR and INSOLU. The pink area of radar plot showed that compound satisfies all the above-mentioned properties except saturation

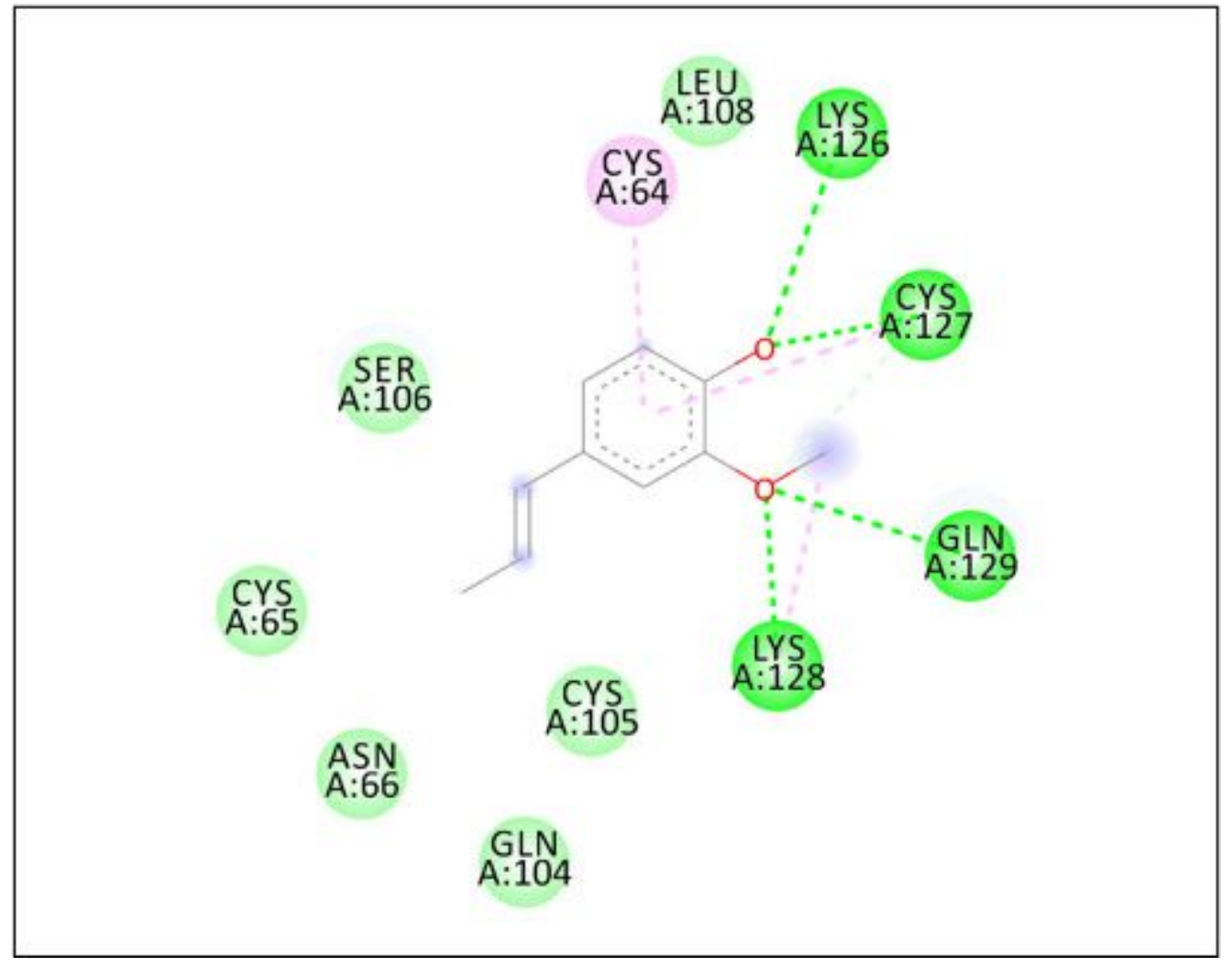

Figure 6 
Binding interactions of isoeugenol with the active site of RodA hydrophobin protein target site (PDB ID: 6GCJ). Green dotted lines depict hydrogen bonds; pink dotted line showed pi-pi bond

\section{Supplementary Files}

This is a list of supplementary files associated with this preprint. Click to download.

- FigureS1.jpg 